\title{
Tonometria de não-contato: variação com medidas repetidas com e sem colírio anestésico
}

\author{
Noncontact tonometry: variation with repeated measures with and without anesthetic \\ eyedrops
}

Ivan Maynart Tavares ${ }^{1}$

Luiz Alberto Soares de Melo Junior ${ }^{2}$

Mário Martins do S. Motta ${ }^{3}$

Fabiana Shinzato Higa ${ }^{4}$

Paulo Augusto de Arruda Mello

Augusto Paranhos Junior ${ }^{6}$
Este trabalho foi realizado no Setor de Glaucoma do Instituto da Visão no Departamento de Oftalmologia da Universidade Federal de São Paulo - (UNIFESP)

Médico Colaborador, Pós-Graduando nível Doutorado.

${ }^{2}$ Médico Colaborador, Preceptor da Residência.

${ }^{3}$ Professor-Titular de Oftalmologia da Faculdade de

Teresópolis, RJ e Professor-Adjunto Doutor de Oftal-

mologia da Faculdade Uni-Rio.

${ }^{4}$ Médica Colaboradora.

${ }^{5}$ Professor-Adjunto Mestre e Doutor, Professor Orientador da Pós-Graduação.

${ }^{6}$ Mestre e Doutor, Chefe do Setor.

Endereço para correspondência: Ivan Maynart

Tavares Rua Botucatu, 822 - São Paulo (SP)

CEP 04023-062 - Fax: (11) 5573-4002

E-mail:imaynart@terra.com.br

Recebido paraanálise em 23.09.2002

Versão revisada recebida em 06.02 .2003

Aprovação em 07.04.2003

\section{RESUMO}

Objetivos: Avaliar a influência da utilização do colírio anestésico, bem como verificar o comportamento da medida da pressão intra-ocular (Po) com a adaptação (aprendizado) do paciente à tonometria de não-contato (TNC). Materiais e métodos: Estudo clínico randomizado duplo-mascarado em que se avaliaram 24 olhos de 24 voluntários normais, sem experiência prévia com a TNC, divididos em 2 grupos: grupo I (TNC com instilação prévia de colírio anestésico seguido de tonometria de aplanação de Goldmann (TAG)) e grupo II (TNC com instilação prévia de colírio placebo seguido de TAG). As medidas da Po foram realizadas em três visitas com intervalo de um a três dias aproximadamente no mesmo horário. Resultados: Constatou-se a Po por meio da TAG como a única variável preditora estatisticamente significante $(\mathrm{p}<0,05)$ para a TNC. Não houve diferença na variação da Po entre os grupos $(\mathrm{p}=0,884)$, tampouco entre as visitas $(p=0,144)$. Houve melhor correlação entre a TNC e TAG no grupo II em relação ao grupo I em todas as visitas, porém esta diferença não se mostrou estatisticamente significante $(p=0,4853)$. Conclusões: A utilização de anestésico tópico e a adaptação de indivíduos normais ao exame de tonometria de não-contato não demonstraram influência significante sobre a medida da Po obtida por este método.

Descritores: Tonometria de não-contato; Tonometria de Goldmann; Pressão intra-ocular; Anestésico; Glaucoma

\section{INTRODUÇÃO}

A medida da pressão intra-ocular (Po) faz parte da propedêutica básica do exame oftalmológico, sendo um dos principais parâmetros para diagnóstico e acompanhamento do glaucoma. A tonometria de aplanação de Goldmann (TAG) é considerada o exame padrão-ouro para a mensuração da $\mathrm{Po}^{(1)}$. A tonometria de não-contato (TNC) foi introduzida por Grolman em 1972:(2) baseando-se no princípio da tonometria de aplanação, o tonômetro possui um sistema pneumático que produz um jato de ar que leva à aplanação da córnea, em cuja superfície é projetado um feixe colimado de luz, em que apenas os raios paralelos e coaxiais refletidos são captados pelo receptor do aparelho, obtendo-se um pico máximo de recepção de luz quando a córnea é aplanada.

O primeiro estudo clínico sobre TNC foi descrito em 1973 por Forbes ${ }^{(3)} \mathrm{e}$ ressaltava suas vantagens, como a aplicabilidade para programas de rastreamento e a não necessidade da utilização de colírios. Outras vantagens apontadas são a realização por pessoal não médico e o menor risco de 
contaminação. Entretanto, Britt e col $^{(4)}$ demonstraram a existência de deiscência do filme lacrimal e formação de microaerosol com o sopro de ar, o que tornaria a TNC não tão asséptica como se imaginava até então.

A TNC geralmente é realizada sem a instilação prévia de colírio anestésico, mas a influência desta substância na medida da Po é controversa ${ }^{(5-7)}$.

Tem sido observado que na TNC são encontrados valores menores da Po na primeira medição, quando comparado às medidas subseqüentes, correlacionando tal fato à ansiedade do paciente (efeito “squeezer") ${ }^{(3,8)}$.

Fatores corneais, como espessura e curvatura ${ }^{(9-10)}$ alteram os resultados tanto da TNC quanto da TAG. Entretanto Paranhos e col $^{(11)}$ demonstraram que a refração e a ceratometria não alteraram a concordância entre os tonômetros estudados.

Este estudo foi elaborado em virtude da utilização cada vez mais freqüente da TNC e de a literatura pertinente ser escassa e controversa em relação ao uso de colírios anestésicos e à existência de efeito aprendizado.

\section{MATERIAIS E MÉTODOS}

Foi realizado um estudo clínico randomizado duplo-mascarado, em que se examinaram voluntários normais, no setor de Glaucoma da Universidade Federal de São Paulo, durante o mês de março de 2002. O estudo foi aprovado pelo Comitê de Ética desta instituição e todos os participantes assinaram termo de consentimento. Os participantes selecionados foram alocados aleatoriamente em dois grupos:

Grupo I - TNC com instilação prévia de colírio anestésico seguida da TAG

Grupo II - TNC com instilação prévia de colírio placebo seguida da TAG

A randomização foi realizada através da geração de números aleatórios por computador. Apenas os olhos direitos foram estudados. Em ambos os grupos realizaram-se as medições da Po em três visitas separadas por um intervalo de um a três dias.

A amostra do estudo constitui-se de voluntários normais, sem história de doença ocular recente ou cirurgia oftalmológica prévia. Foram excluídos os indivíduos que apresentavam alterações significativas do segmento anterior ocular à biomicroscopia, bem como os que relataram experiência prévia com a TNC.

O exame oftalmológico realizado na primeira visita constou de medida da acuidade visual corrigida, ceratometria (Kt) computadorizada (Topcon KR-8000), biomicroscopia do segmento anterior e exame de tonometria. Nas duas visitas subseqüentes foram realizadas apenas as tonometrias. As três visitas foram realizadas aproximadamente no mesmo horário (entre 12 e 14 horas).

Para a realização das tonometrias foram utilizados o tonômetro de não-contato da Topcon (CT-80) e o tonômetro de Goldmann (calibrado antes do início do estudo) acoplado à lâmpada de fenda. Inicialmente foi realizada a TNC (com instilação prévia de colírio anestésico ou placebo, a depender do grupo de alocação) e utilizou-se a média de três medidas consecutivas para a análise. Após 15 minutos da TNC, realizava-se a TAG, adotando-se a média de 2 medidas consecutivas para o estudo. Foi adotado o intervalo de 15 minutos em virtude que medidas sucessivas em curtos intervalos de tempo poderem acarretar em medidas imprecisas da Po. ${ }^{(12)} \mathrm{A}$ instilação do colírio anestésico ou placebo imediatamente antes da realização da TNC era realizada por um pesquisador que não efetuava as tonometrias e era o único indivíduo com conhecimento do grupo o qual os voluntários pertenciam. Um examinador realizava a TNC e um segundo, a TAG sem conhecimento dos valores prévios da medida da Po. Os colírios utilizados no estudo foram o cloridrato de proximetacaína 0,5\% (Visonest $^{\circledR}$, Allergan) como anestésico, o cetorolac de trometamina 0,5\% (Cetrolac ${ }^{\circledR}$, Genom) como placebo, e a fluoresceína sódica (Allergan).

Para a análise estatística foram utilizados o teste $\mathrm{t}$ de Student para amostras independentes e o teste exato de Fisher para a comparação, respectivamente, das variáveis numéricas e categóricas dos dados dos participantes entre os 2 grupos de estudo. Para a análise dos dados referentes à medida da Po foram utilizados a regressão linear múltipla para cada uma das 3 visitas a fim de avaliar-se a influência dos grupos, da ceratometria e da TAG em relação à TNC; a análise de variância (ANOVA) de medidas repetidas com dois critérios (grupos e visitas); o teste de correlação intraclasse e o teste para homogeneidade de coeficiente de correlação para avaliar a relação entre a TNC e a TAG e as visitas. Em todos os testes foi adotado 0,05 bi-caudal como nível de significância. Para o cálculo do tamanho da amostra foi adotado $1 \mathrm{mmHg}$ como uma diferença clínica relevante da pressão intra-ocular tanto para variação entre os grupos bem como entre as visitas; 0,05 como nível de significância estatística e 80\% como poder mínimo do teste. Através destes parâmetros calculou-se que 2 grupos de 6 indivíduos atingiriam um poder de teste de $88 \%$ para detectar-se uma diferença de $1 \mathrm{mmHg}$ (desvio-padrão de 0,86 mmHg) entre os grupos e um poder de teste de $83 \%$ para detectar-se uma diferença de $1 \mathrm{mmHg}$ (desvio-padrão de $0,37 \mathrm{mmHg}$ ) entre as visitas e interação entre os grupos e as visitas.

\section{RESULTADOS}

Foram selecionados 24 indivíduos normais para o estudo. Onze indivíduos foram alocados aleatoriamente para o grupo I e 13 indivíduos para o grupo II. Os dados demográficos encontram-se na tabela 1. Verifica-se que houve uma distribuição homogênea entre os grupos. As medidas de Po realizadas através da TNC e TAG encontram-se na tabela 2. A figura 1 apresenta um gráfico do tipo "box plot" destas medidas.

Através do teste de regressão linear múltipla para cada uma das três visitas, utilizando-se como variável dependente a Po através da TNC e como variáveis independentes a Po por 
meio da TAG, a alocação do grupo, a Kt média e a diferença de Kt, constatou-se a Po através da TAG como a única variável preditora estatisticamente significante $(\mathrm{p}<0,05)$ da Po através da TNC.

Em relação às medidas repetidas da diferença de Po entre a TNC e TAG, não existiu interação entre as visitas e os grupos ( $p=0,906)$, bem como não houve diferença entre os grupos $(p=0,884)$ tampouco entre as visitas $(p=0,144)$ (Figura 2$)$.

Na tabela 2 encontram-se os coeficientes de correlação intraclasse (r) de acordo com o grupo e a visita. Houve uma melhor correlação da TNC com a TAG no grupo II em relação ao grupo I em todas as visitas, porém esta diferença não se mostrou estatisticamente significante $(p=0,4853)$ através do teste de homogeneidade de coeficiente de correlação.

\section{DISCUSSÃO}

No presente estudo encontrou-se uma correlação estatisticamente significante da TNC com a TAG, fato amplamente descrito na literatura ${ }^{(13-14)}$. Ressalta-se que há diferenças na confiabilidade entre os diversos aparelhos empregados para a TNC. ${ }^{(13,15)}$ Comparando a TNC com a TAG, Popovich e col ${ }^{(16)}$ encontraram uma diferença com desvio-padrão de 2,3 mmHg para a população geral do estudo e de 3,3 mm Hg para o grupo com pressão maior que $20 \mathrm{mmHg}$. Moseley e col $^{(17)}$ observaram uma variação de $\pm 3 \mathrm{mmHg}$ em $79 \%$ dos casos, enquanto Brencher e col ${ }^{(18)}$, usando o Topcon CT-10, demonstraram que no grupo com Po entre 10 e 20 mmHg a TNC foi consistentemente mais alta que a TAG, dentro de um limite de $4 \mathrm{~mm} \mathrm{Hg}$. Calixto

\begin{tabular}{|c|c|c|c|}
\hline & Grupo I & Grupo II & $p$ \\
\hline n (\%) & $11(45,8 \%)$ & $13(54,2 \%)$ & \\
\hline Idade (anos) & $34,3 \pm 12,4$ & $28,5 \pm 7,8$ & 0,177 \\
\hline Sexo M:F & $5: 6$ & $4: 9$ & 0,675 \\
\hline Raça & & & 0,386 \\
\hline caucasiana & 9 & 8 & \\
\hline negra & 2 & 3 & \\
\hline amarela & 0 & 2 & \\
\hline \multicolumn{3}{|l|}{ Acuidade visual } & 0,222 \\
\hline logMAR / Snellen & 0,045 / 20/20 & $0,008 / 20 / 20$ & \\
\hline Kt média (D) & $42,672 \pm 1,488$ & $43,878 \pm 2,552$ & 0,070 \\
\hline Diferença Kt (D) & $0,633 \pm 0,610$ & $0,874 \pm 0,265$ & 0,305 \\
\hline \multicolumn{4}{|c|}{$\mathrm{M}=$ masculino; $\mathrm{F}=$ feminino; $\mathrm{Kt}=$ ceratometria $; \mathrm{D}=$ dioptria } \\
\hline
\end{tabular}

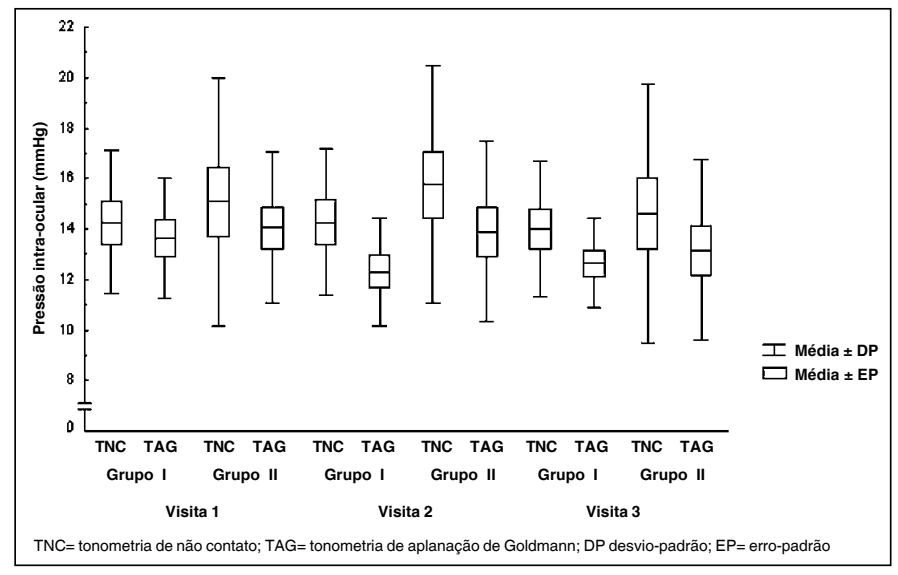

Figura 1 - Pressão intra-ocular mensurada através da TNC e TAG em relação aos grupos e visitas

e col $^{(6)}$ encontraram valores mais elevados com a TNC em relação a TAG quando Po até $30 \mathrm{mmHg}$, mas valores mais baixos quando a TAG foi maior que $30 \mathrm{mmHg}$. Shinzato ${ }^{(9)}$ também relatou que as medidas da TNC foram significantemente maiores que as da TAG. Gus e col ${ }^{(19)}$ e Koopmans e col $^{(20)}$ sugerem que medidas acima de $20 \mathrm{mmHg}$ na TNC sejam confirmadas pela TAG, para se evitarem decisões clínicas errôneas.

A TNC normalmente é realizada sem a instilação prévia de anestésico. Kass $^{(7)}$ descreve que há uma melhor acurácia deste método quando se instila colírio anestésico, sem apresentar fundamentos para tal assertiva. Já Calixto e col $^{(6)}$ não comprovaram a influência do uso de anestésico na TNC, o que corrobora o resultado encontrado no presente estudo. Baundouin $^{(5)}$ ressalta o fato de alguns anestésicos poderem reduzir a Po farmacologicamente, facilitando a drenagem do humor aquoso, e especula que este fato pode ser responsável pelas diferenças entre a TNC, normalmente sem anestesia, e TAG, sempre com anestesia.

Neste estudo encontrou-se uma correlação maior no grupo II (placebo) em relação ao grupo I (anestésico) entre a TNC e a TAG, apesar de não ter sido estatisticamente significante. Não foi encontrada literatura pertinente a este tópico.

Não foi verificada alteração nos resultados das medidas da Po através da TNC em medidas sucessivas. Em sua primeira publicação sobre a TNC, Forbes ${ }^{(3)}$ alertava para o chamado efeito "squeezer", no qual a primeira medida da Po mostravase menor que as seguintes devido à ansiedade do paciente com um conhecimento prévio do exame, fato também descrito por Vernon. ${ }^{(8)}$

\begin{tabular}{|c|c|c|c|c|c|c|c|c|c|}
\hline & \multicolumn{3}{|c|}{ Visita 1} & \multicolumn{3}{|c|}{ Visita 2} & \multicolumn{3}{|c|}{ Visita 3} \\
\hline & TNC & TAG & $r$ & TNC & TAG & $\mathbf{r}$ & TNC & TAG & $\mathbf{r}$ \\
\hline Grupo I & $14,27 \pm 2,83$ & $13,64 \pm 2,36$ & 0,38 & $14,28 \pm 2,90$ & $12,32 \pm 2,14$ & 0,46 & $14,00 \pm 2,68$ & $12,63 \pm 1,77$ & 0,38 \\
\hline Grupo II & $15,08 \pm 4,91$ & $14,04 \pm 2,99$ & 0,76 & $15,78 \pm 4,73$ & $13,88 \pm 3,58$ & 0,84 & $14,62 \pm 5,16$ & $13,15 \pm 3,57$ & 0,70 \\
\hline
\end{tabular}




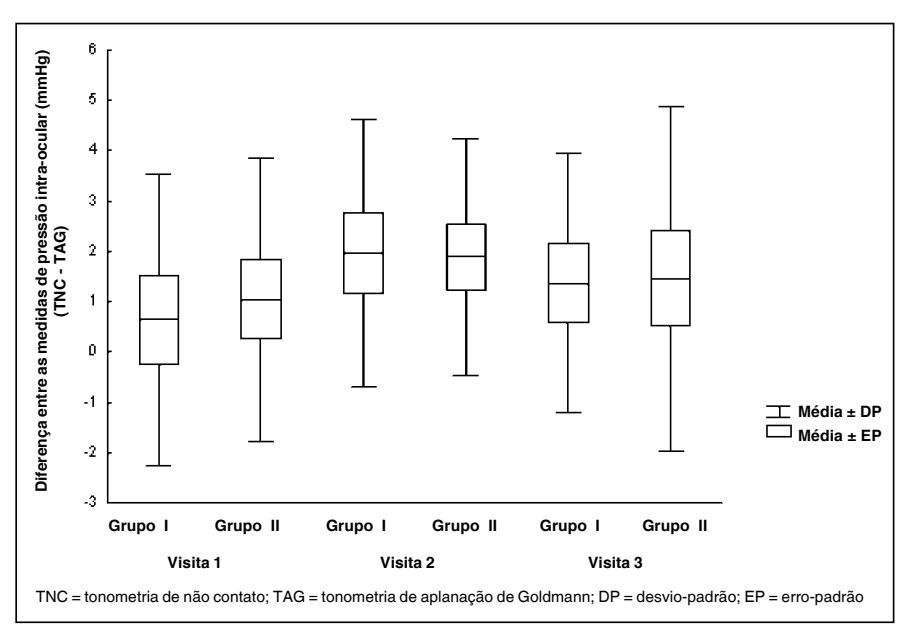

Figura 2 - Diferenças entre as medidas de pressão intra-ocular realizadas através da TNC e TAG em relação aos grupos e visitas

Uma limitação do presente estudo é o tamanho da amostra, o que provoca uma diminuição do poder dos testes estatísticos para verificarem-se variações muito pequenas de pressões intra-oculares, no entanto, o tamanho da amostra é maior do que o necessário para detectar-se variações maiores ou iguais a $1 \mathrm{mmHg}$, isto é, valores que poderiam ser considerados relevantes do ponto de vista clínico. A amostra constituída por apenas indivíduos normais impossibilitou a análise do comportamento de variáveis como a Po em situação de valores extremos.

Em conclusão, neste estudo a utilização de anestésico tópico e a adaptação de indivíduos normais ao exame de tonometria de não-contato não demonstraram influência estatisticamente significante sobre a medida da Po obtida através deste método.

\section{ABSTRACT}

Purpose: To evaluate the influence of the previous instillation of anesthetic eye drops, as well as to verify intraocular pressure (IOP) performance with adaptation to noncontact tonometry (NCT). Methods: A randomized double-masked study in which 24 eyes of 24 normal volunteers were studied, divided into group I (NCT with anesthetic instillation) and group II (NCT with placebo instillation). Subjects in both groups were submitted to Goldmann applanation tonometry (GAT) after NCT. IOP was measured on three different visits, with an interval of 1 to 3 days. Results: IOP measured with GAT was the only statistically significant predictor variable $(\mathrm{p}<0.05)$ for NCT. There was no difference in IOP variation between the groups $(\mathrm{p}=0.884)$ or between the visits $(\mathrm{p}=0.144)$. A better correlation was found between GAT and NCT in group II (placebo) when compared to group I (anesthetic) on all visits, but this difference was not statistically significant $(\mathrm{p}=0.4853)$. Conclusions: Previous instillation of anesthetic eye drops and normal adaptation of normal individuals to NCT did not demonstrate a significant influence on IOP measures.

Keywords: Noncontact tonometry; Goldmann tonometry; Intraocular pressure; Anesthetic; Glaucoma

\section{REFERÊNCIAS}

1. Whitacre MM, Stein R. Sources of error with use of Goldmann-type tonometers. Surv Ophthalmol 1993;38:1-30.

2. Grolman B. A new tonometer system. Am J Optom Arch Am Acad Optom 1972;49:646-60.

3. Forbes M, Pico Jr. G, Grolman B. A noncontact applanation tonometer. Sight Sav Rev 1973;43:155-61.

4. Britt JM, Clifton BC, Barnebey HS, Mills RP. Microaresol formation in noncontact "air-puff” tonometry. Arch Ophthalmol 1991;109:225-8.

5. Baundouin C, Gastaud P. Influence of topical anesthesia on tonometric values of intraocular pressure. Ophthalmologica 1994;208:309-13.

6. Calixto N, Cronemberger S, Calixto JM, Antunes CS. Estudo comparativo entre a tonometria de não-contato com o tonômetro Pulsair e a tonometria de aplanação de Goldmann. Rev Bras Oftal 1994;53:9-15.

7. Kass MA. Standardizing the measurement of intraocular pressure for clinical research. Ophthalmology 1996;103:183-5.

8. Vernon SA. Reproducibility with the Keeler Pulsair 2000 non-contac tonometer. Br J Ophthalmol 1995;79:554-7.

9. Shinzato GT. Avaliação da pressão intra-ocular com os tonômetros de nãocontato, Tono-pen e Goldmann em olhos submetidos a LASIK para correção refrativa de miopia e astigmatismo miópico [tese]: Belo Horizonte, Universidade Federal de Minas Gerais; 2000.

10. Recep OF, Hasiripi H, Cagil N, Sarikatipoglu H. Relation between corneal thickness and intraocular pressure measurements by noncontact and applanation tonometry. J Cataract Refract Surg 2001;27:1787-91.

11. Paranhos Jr A, Paranhos F, Prata Jr JA, Omi CA, Mello PAA, Shields MB. Influence of keratometric readings on comparative intraocular pressure measurements with Goldmann, Tono-Pen and noncontact tonometers. J Glaucoma 2000;9:219-23.

12. Recep OF, Hasiripi H, Vayisoglu E, Kalayci D, Sarikatipoglu H. Accurate time interval in repeated tonometry. Acta Ophthalmol Scand 1998;76:603-5.

13. Wingert TA, Bassi C, McAlister WH, Galanis JC. Clinical evaluation of five portable tonometers. J Am Optom Assoc 1995;66:670-4.

14. Yamane Y, Yamane R. Avaliação da pressão intra-ocular pela tonometria de não-contato. Rev Bras Oftal 1994;53:11-7.

15. Moreno-Montanés J, Gómez-Demmel E, Lajara-Blesa J, Aliseda-Perez de Madrid D. Comparative study of three non-contact tonometers and the Goldmann tonometer. Ophthalmologica 1994;208:115-8.

16. Popovich KS, Shields MB. A comparison of intraocular pressure measurements with the XPERT noncontact tonometer and Goldmann applanation tonometry. J Glaucoma 1997;6:44-6.

17. Moseley MJ, Thompson J, Deutsch J, Misson GP, Naylor G, Tan-Yee A, Taylor RH, Fielder AR. Comparison of the Keeler Pulsair 2000 non-contact tonometer with Goldmann applanation. Eye 1993;7(Pt 1):127-30.

18. Brencher HL, Kohl P, Reinke AR, Yolton RL. Clinical comparison of air-puff and Goldmann tonometers. J Am Optom Assoc 1991;62:395-402.

19. Gus PI, Sklaude PBV, Kosmalski VS, Gerstner AP, Kwitko IL, Kwitko S. Estudo comparativo entre a tonometria de aplanação (Perkins) e a tonometria de não-contato (Topcon CT-20) em olhos glaucomatosos e normais. Arq Bras Oftal 1998;61:70-3.

20. Koopmans SA, Kooijman AC, van Rij G, Eisses J, Groot-Woltjer J. Clinical evaluation of two non-contact tonometers. Doc Ophthalmologica 1991; 78:259-63. 